\title{
A Near-Field-based TPMS Solution for Heavy Commercial Vehicle Environement
}

\author{
Ahmad Rida Tawakuli \\ Secan-Lab \\ University of Luxebmourg \\ Luxembourg \\ ahmad.tawakuli@uni.lu
}

\author{
Ridha Soua \\ IEEE member \\ Luxembourg \\ ridha.soua@gmail.com
}

\author{
Thomas Engel \\ Secan-Lab \\ University of Luxembourg \\ Luxembourg \\ thomas.engel@uni.lu
}

\begin{abstract}
This paper proposes a Near-Field Communication (NFC) based solution for Tire pressure monitoring system (TPMS) in heavy commercial vehicles instead of the high frequency Far-Field communication used in conventional TPMS. We simulate the application environment represented by the tire and wheel combination, and the proposed system transmitter to evaluate the signal propagation using finite element method (FEM) analysis; incorporating highly detailed models of the transmitter and the wheel and tire combination. The simulation demonstrates the adverse effect of the application environment on the signal propagation, and shows the merit of using NF-based communication compared to conventional state of the art TPMS solutions.
\end{abstract}

Keywords- Near-field, TPMS, FEM, heavy commercial vehicle, truck, $\mathrm{RF}$, propagation, antennas, automotive, tires, sensors.

\section{INTRODUCTION}

Despite its first use more than 120 years ago, Near-Field communication (NF) was superseded soon after by Far-Field communication (FF) due to the later's longer range and versatile applications. NF was soon forgotten as RF engineers and Antenna system developers continued to develop higher frequencies FF communication to provide larger bandwidth and data transfer speeds. Recent Automotive applications of $\mathrm{NF}$ communication and similarly inductive power transfer have brought NF back into the spotlight due to its higher efficient power consumption, secure and robust communication in harsh environments such as around metals $[1,2,3]$.

Tire Pressure Monitoring System (TPMS) for heavy commercial vehicles will become a mandatory safety equipment, as EU regulations mandate that all commercial vehicles be equipped with TPMS by 2022 and all TractorTrailer vehicles by 2024 [4]. Conventional TPMS solutions, which use the electromagnetic waves generated by the oscillating current in the antenna, ie. Far-Field communication, are seeing limited success $[5,6]$ and their applications are reduced to smaller and lighter commercial vehicles. Therefore, efforts have been stepped up in the past few years to develop reliable TPMS (reliable signal propagation) in harsh environments such as heavy commercial vehicles. A previous study [7] detailed how current state of the art TPMS do not provide an efficient solution for the TractorTrailer heavy commercial vehicles, the most common type of vehicles used by logistics fleets. The authors have demonstrated RF signal attenuation that conventional TPMS suffers from in heavy commercial vehicles, by conducting a Finite Element Analysis (FEM) of an actual commercial vehicle TPMS Solution [8], applied to a highly detailed wheel and tire model. The RF propagation of the transmitter unit has been calculated as accurately as possible for this complex multilayer and multi material structure of the tire [9], which have different electrical and magnetic properties [10].

In this paper, we leverage on NF communications to model a near-field-based TPMS transmitter unit. NF is a technology with growing applications in the automotive sector $[1,3]$ and contrarily to Far-Field communication, it uses the electric fields $(H$-Field) and the Magnetic field ( $B$-field) generated around the Antenna just before the fields merge to form the Electromagnetic waves. The fields from two communication antennas interact via inductive coupling forming the base of NF communication [11] as illustrated in Figure 1 [12]. The strength of these fields varies depending on the antenna type, while the range, in which the fields are present, varies depending on the operating frequency [13]. H-Field and BField present high decay rates, short detection ranges and show robustness in metal environments. This makes NF a suitable communication solution for heavy commercial vehicles $[14,15]$.

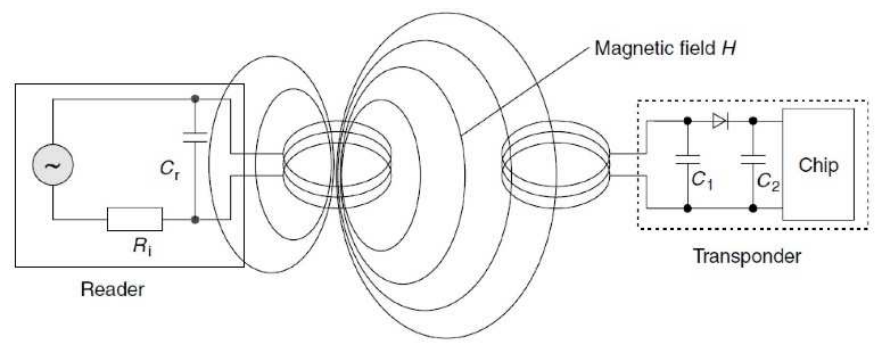

Figure 1:Inductive Coupling between the transmitter and receiver antennas in NF Communication [12]

In this paper, we conduct an accurate evaluation of NF propagation in a heavy commercial vehicle environment using a highly detailed model of an actual heavy commercial vehicle tire and wheel. The remainder of this paper is organized as follow: Section II evaluates NF propagation in a free space; and will be considered as a baseline measurement. Section III evaluates NF propagation inside the wheel and the tire of a truck, and compares the results to the conventional TPMS propagation [7]. Section IV concludes the paper.

\section{EVALUATION OF RF PROPAGATION IN FREE-SPACE}

\section{A. Modeling of the NF-based TPMS Transmitter unit}

To have a benchmark for comparing NF propagation in the truck tire and wheel combination, we first created a detailed model of the transmitter unit using similar dimensions and power supply to a state-of-the-art TPMS sensor [8]. We use the Ansys Maxwell (Low-frequency simulator) electromagnetics suite [16] to design the transmitter and analyze the field propagation. Commercial NF systems such as NFC uses loop antennas to generate predominantly 
Magnetic $B$-Fields [11]. In this paper, we adopt a similar antenna principle but using the low operating frequency of 125 $\mathrm{kHz}$ for optimum the field range. An additional advantage is that the transmitter will operate on the European TPMS standard frequency range of $125 \mathrm{kHz}$ used in some conventional TPMS sensors for wake-up signals. Thus, our proposed setup meets the EU TPMS standard [17].

The transmitter comprises of an integrated circuit with a temperature sensor, a pressure sensor and the transmitting antenna. Our model of the transmitter consists of a PCB board which may include the temperature and pressure sensors, the integrated circuit (IC) unit, the power unit, and the transmitting antenna. Due to the size constrain and the need for high transmission efficiency, we use a ferrite rod antenna. This type of antenna allows high inductance values compared to the antenna size. For the selected frequency $(125 \mathrm{kHz})$, an inductance of around $135 \mu \mathrm{H}$ is required. A detailed model of the transmitter is shown in Figure 2.

The wheel dimensions and structure as well as sensor mounting position were taken into consideration when designing the transmitter antenna. To maximize the antenna inductance hence the generated field strength, we select the longest length possible for the ferrite rod: $4 \mathrm{~cm}$ long core allows for a high loop number while fitting within the wheel barrel mid-section recess. This fitting position will make the transmitter non-intrusive when mounting and dismounting tires, therefore preventing damage to the transmitter. The transmitter design also takes into consideration the IC and PCB board as well as battery unit. The sensor is encased by a hard plastic mold 3-4 mm thick, which also insulates the transmitter from the wheel. The transmitter could be fastened to the wheel barrel in a similar way to conventional TPMS sensors detailed in [7].

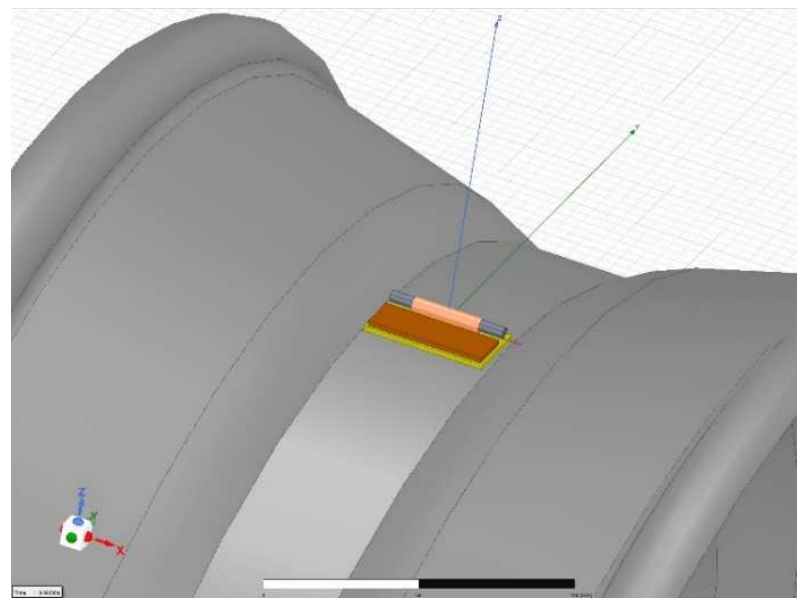

Figure 2: Transmitter unit design and position used in the FEM Analysis

\section{B. Analysis of RF propagation in free space}

Our analysis uses the actual transmitter design parameters (see Table 1) rather than theoretical design parameters. The incident power takes into consideration the interference regulations and the sensor battery life. Parameters are highlighted in Table 1. The analysis requires a field propagation region to be defined, in which the software would calculate the fields generated. To demonstrate the field propagation and field strength in free space in comparison to the wheel and tire volume, we have included the wheel and tire as non-active models in the propagation figures but not in the actual analysis. We have also assigned a free-space analysis region similar in dimensions to the wheel and tire combination, which was around 1.7 cubic meter.

Table 1: Antenna and Analysis parameters

\begin{tabular}{|l|l|}
\hline \multicolumn{1}{|c|}{ Parameter } & \multicolumn{1}{c|}{ Value } \\
\hline Incident Current & $3.3 \mathrm{~mA}$ via coil terminal \\
\hline $\begin{array}{l}\text { Specific Operating } \\
\text { Frequency }\end{array}$ & $\begin{array}{l}125 \mathrm{kHz} \text { (included in EU } \\
\text { TPMS standard) }\end{array}$ \\
\hline Port impedance & $\begin{array}{l}\text { Matching network with } \\
\text { varying capacitance value } \\
\text { in the Pico farad range. }\end{array}$ \\
\hline Antenna type & Ferrite loop antenna \\
\hline Antenna material & Copper with Ferrite core \\
\hline PCB Material & FR4 \\
\hline Analysis result shown in Figures 3,4 and 6 display a fairy
\end{tabular}

Analysis results shown in Figures 3, 4, 5 and 6 display a fairly omni-directional field propagation across the analysis region, with a gradual reduction of the field strength as it moves farther away from the antenna. The field propagates from one end of the antenna to the other in a semi-circular movement as expected for ferrite core antennas. A slight field distortion towards the $+y$ and $+z$ direction away from the $\mathrm{PCB}$ can be attributed to the antenna ground plane. The Antenna also shows the characteristics magnetic loop antenna [11] in which the H-Field in very small. B-Field ranges from 1199 mGauss around the antenna coils to $0.15 \mathrm{mGauss}$ around the edge of the region. The $\mathrm{H}$-field ranges from 95 Henries near the core, down to 0.0095 Henries near the edge of the analysis region. The propagation pattern in free spaces is illustrated as field vector plots in Figure 3 and Figure 4 for the B-field and Figure 5 and Figure 6 for the H-Field. The plots present the propagation relative to the non-active wheel and tire models, which were included for visualization purposes but not considered in the free space analysis.

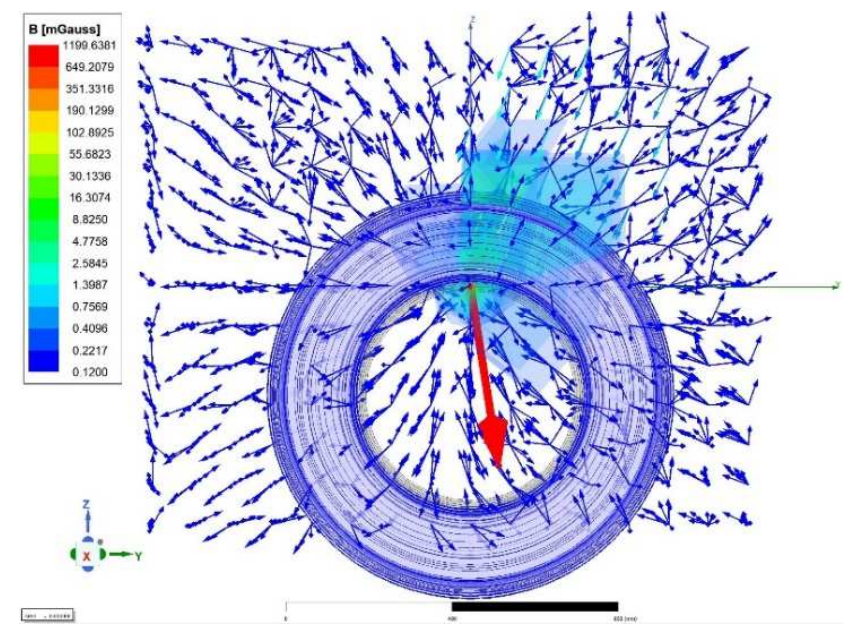

Figure 3: Propagation of the B-field in free space along the YZaxis relative to the wheel/tire model 


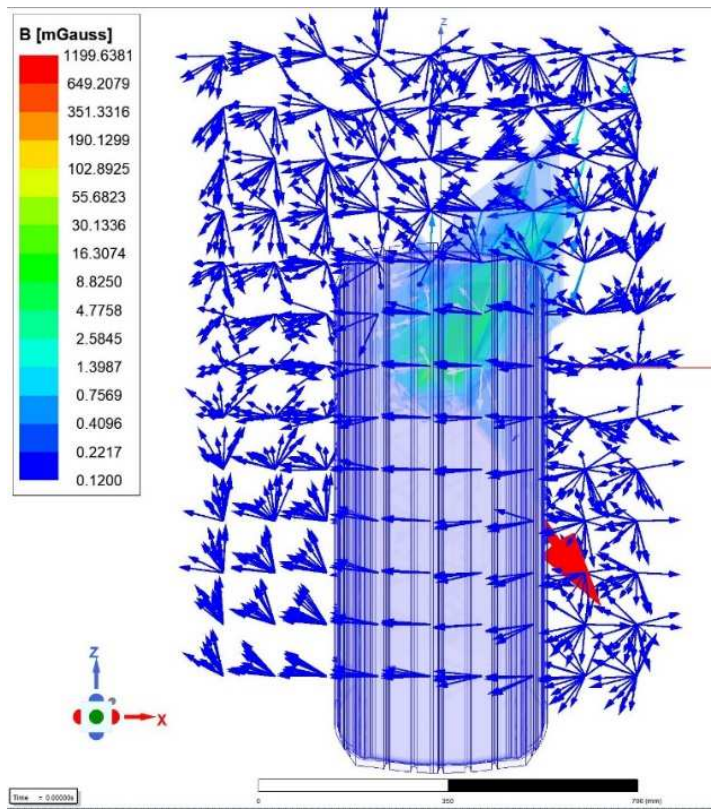

Figure 4: Propagation of the B-field in free space along the XZaxis relative to the wheel/tire model

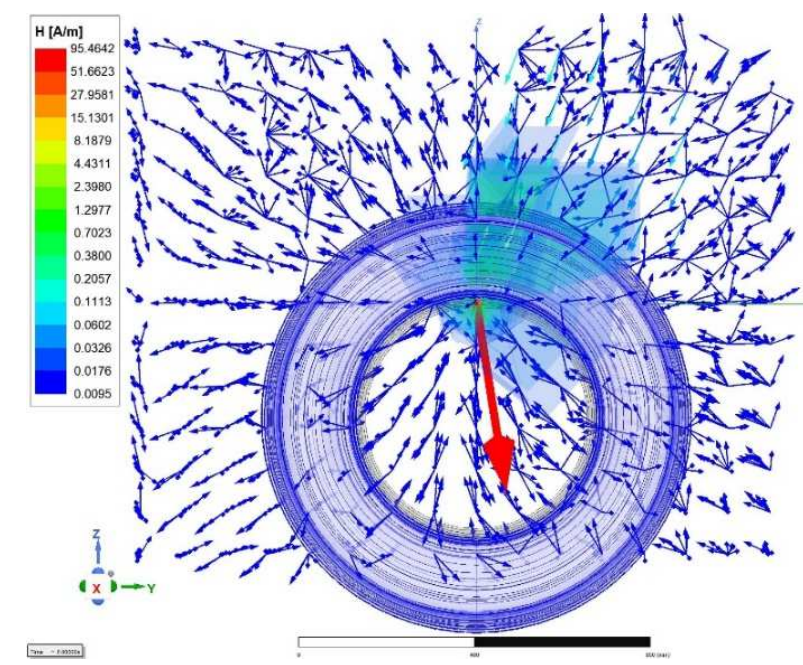

Figure 5: Propagation of the H-field in free space along the YZaxis relative to the wheel/tire model

\section{EVALUATION OF RF PROPAGATION IN A HEAVY VEHICLE ENVIRONMENT}

\section{A. Modelling a commercial vehicle tire and wheel}

Before starting the complex FEM analysis of NF propagation inside the wheel and tire, we have to determine the main cause of signal attenuation. Previous FEM analysis represented the tire as a single structure comprising of a single material (i.e. Rubber) $[18,19,20]$. However, tires are a combination of different materials with a multi-layer structure. In our previous study [7], we described the commercial tire internal structure and we outlined the differences from passenger car. Heavy vehicle tires present much greater challenges, as the carrying load requirements are much greater than passenger cars, hence the need for greater structural reinforcement of the tire.

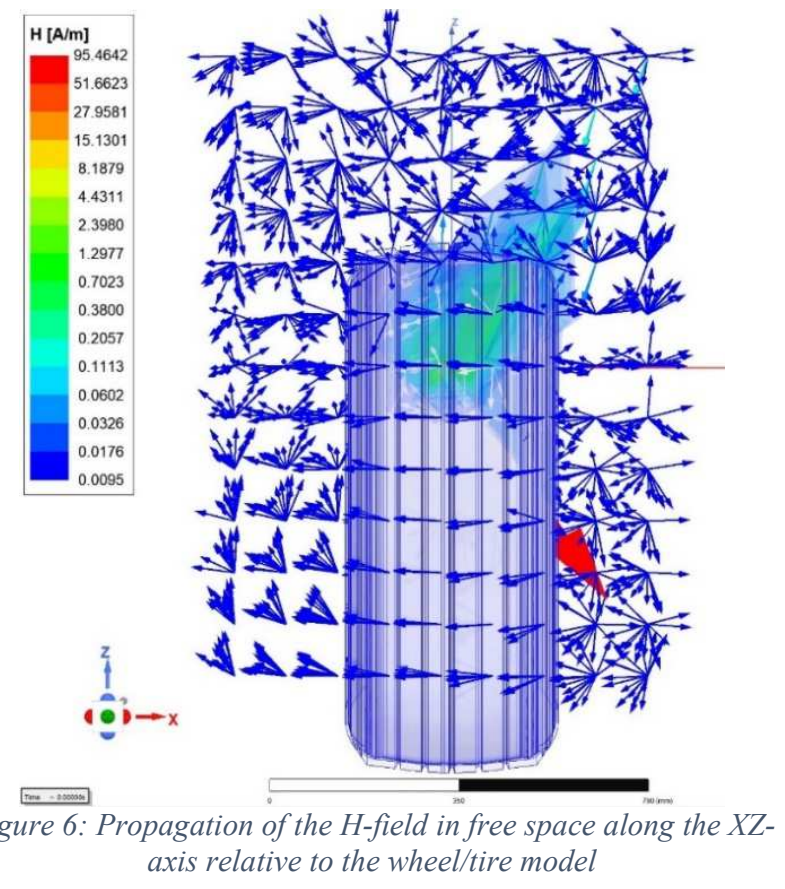

In this paper, we use the same 3D tire and wheel combination model, which was used to test the conventional TPMS in [7]. These are current production models and are used for long haul tractor-trailers and are modelled in actual size. The commercial vehicle tire consists of around 54 different components and layers with some components overlapping. This presents a challenge for the analysis and mesh building in Ansys maxwell as the different equations used in this modeling uses magnetic properties of the material. Hence, the tire and wheel models have to be modified to merge some components of similar material in order to complete the meshing process successfully. We modify a tire model to reflect the various factors mentioned previously and to make the magnetic properties, such as relative permeability, more uniform. We integrate the wheel design into the model with actual specifications of the steel wheel of 9 inch width and 22.5 inch diameter commonly used in European tractortrailer in the model. The wheel sits flush with the tire chafer. In actual fitting, the side walls of the tire are protruding beyond the wheel and the tire chafer is pushing into the rim flange to create an air tight seal. This cannot be modelled as it depends on tire pressure and side wall flexing in relation to the tread contact point with the ground and several other factors. The overall diameter of the model is $984 \mathrm{~mm}$ across, and the width is $400 \mathrm{~mm}$

\section{B. Analysis of NF propagation inside the tire and wheel}

In order to analyze NF propagation in the commercial vehicle environment, we place the transmitter model inside the tire carcass. The transmitter is placed similarly to conventional TPMS: i.e. on top of the middle section of the wheel barrel. The TPMS sensors are insulated and ruggedized by encasing them sensor in a plastic mold. The encasing was not modelled, but the transmitter was insulated from the wheel barrel by a layer of glass fiber reinforced plastic of relative permittivity 12 . Once the model is complete, we set the sweep setting, incident power and excitation parameters. The analysis also requires an evaluation region to be defined. This is the region where the Electromagnetic fields are evaluated. 
The region was set at around 3 times the model size, giving an evaluation area around 10 cubic meters.

The propagation pattern of the Antenna fitted inside the wheel/tire model is illustrated as magnetic field vector plots in Figure 7 and Figure 8 for the B-field. The figures show the propagation with the actual sealed Wheel and Tire model considered in the analysis.

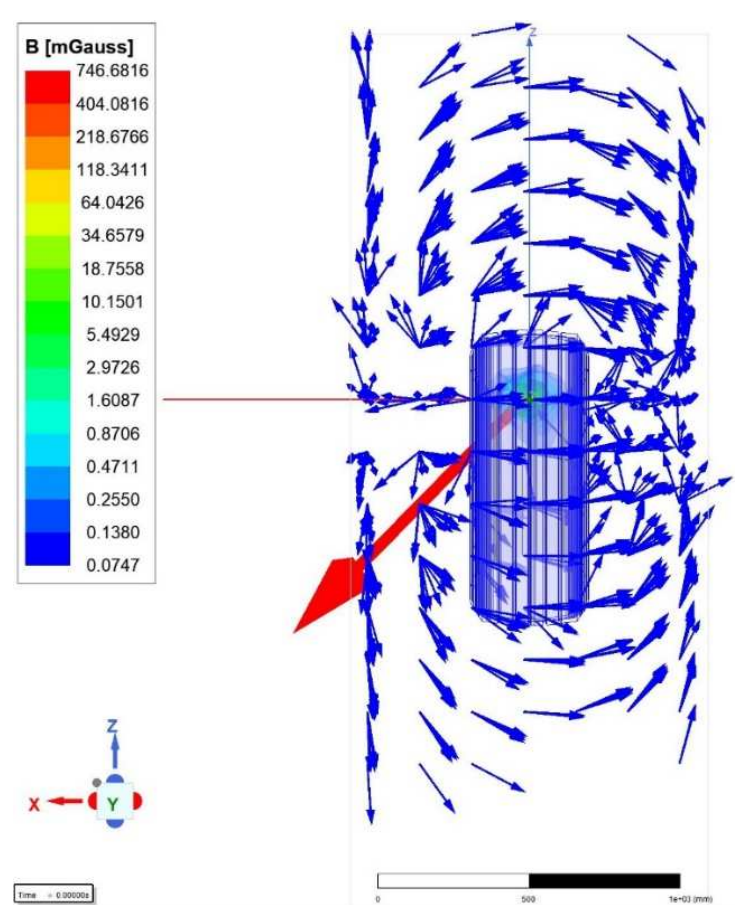

Figure 7: Propagation of the B-field along the X-axis and Z-axis with the wheel/tire model

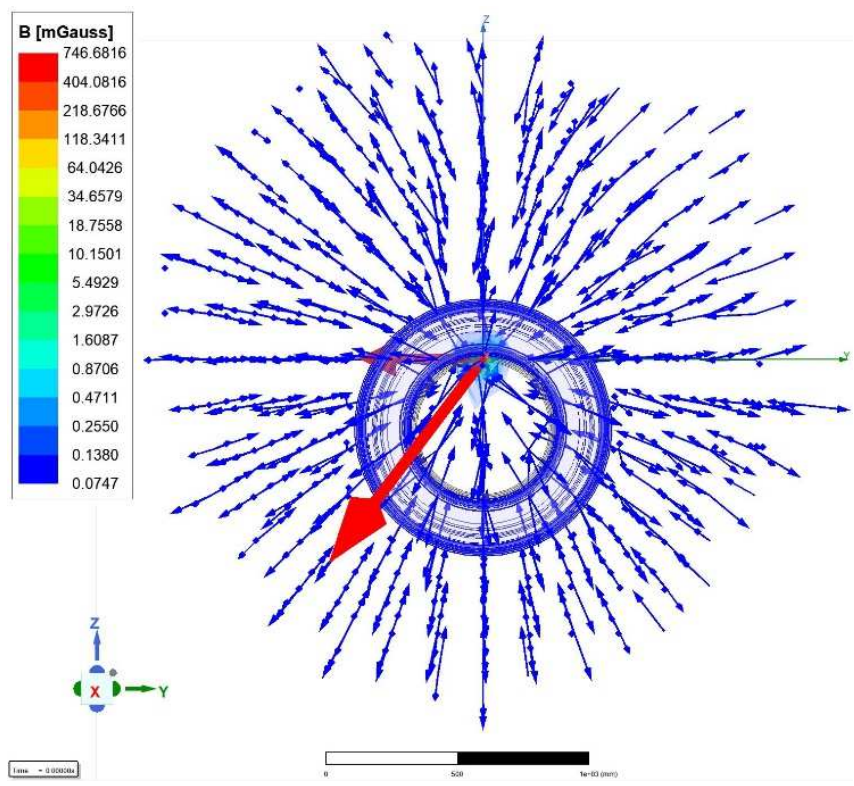

Figure 8: Propagation of the B-field along the Y-axis and Z-axis with the wheel/tire model

The Antenna maintains its isotropic characteristics to a large extent and the field propagation is omni-directional. The $B$ Field propagated through the tire tread and extended partially the wheel barrel. The fields strength has been reduced by around $38 \%$ while the maximum magnetic field strength has been reduced to 746.6 mGauss. The minimum magnetic field strength has been also reduced by 0.074 mGauss in the given simulation region. The wheel barrel also channels the radiating fields along the barrel, which explains this omnidirectional propagation. Our modeling takes into account the complete structure of the wheel and tire combination. That is why it can be seen that the field pattern is maintained, allowing for field detection in all directions. The thick steel sheets of the wheels has attenuated the field to some extent, while the multiple layers of steel mesh do not appear to have significantly attenuated the field. These results show a significant improvement to the greatly distorted and attenuated propagation seen in Figures 9 and Figure 10 of the conventional TPMS at a significantly less transmission power. Unlike in conventional TPMS, the NF solution has no apparent null points in the radiation due to the surrounding structures, nor is the radiation completely shielded by any of the structures (see Figure 7 and Figure 8). The fields shape is not severely distorted and the radiation is still symmetrical to a large extent. While the maximum propagation range of the TPMS antenna is around 1.4 meters to 1.8 meters at the largest lobe, the side lobes are significantly smaller and the propagation range is less in these lobes (see Figure 9 and Figure 10). In the proposed NF system, the maximum field propagation in the given free space is similar to the TPMS system at around 1.5 meters, but is more symmetrical (see Figure 7 and Figure 8). These characteristics could reduce the number of receiver antennas due to the more efficient field detection, which will reduce the required infrastructure in both the tractor and trailer, hence reducing overall costs.

The field propagation in the NF system could be higher if higher transmission power is used. It is worth noting that the free space region used in the analysis could be enlarged to provide the field strength in a larger volume. The later could not be examined due to the higher computational power required and substantially long analysis time.

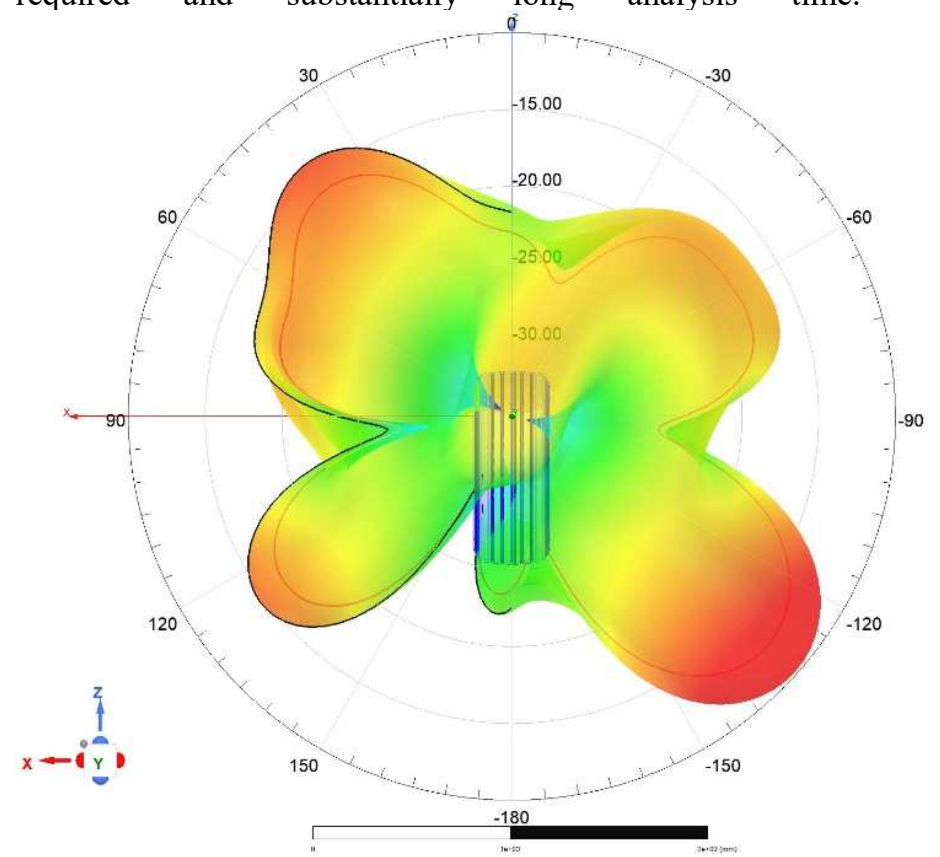

Figure 9: Propagation pattern along the $X$ and $Z$ axis with the Tire and Wheel in conventional TPMS 


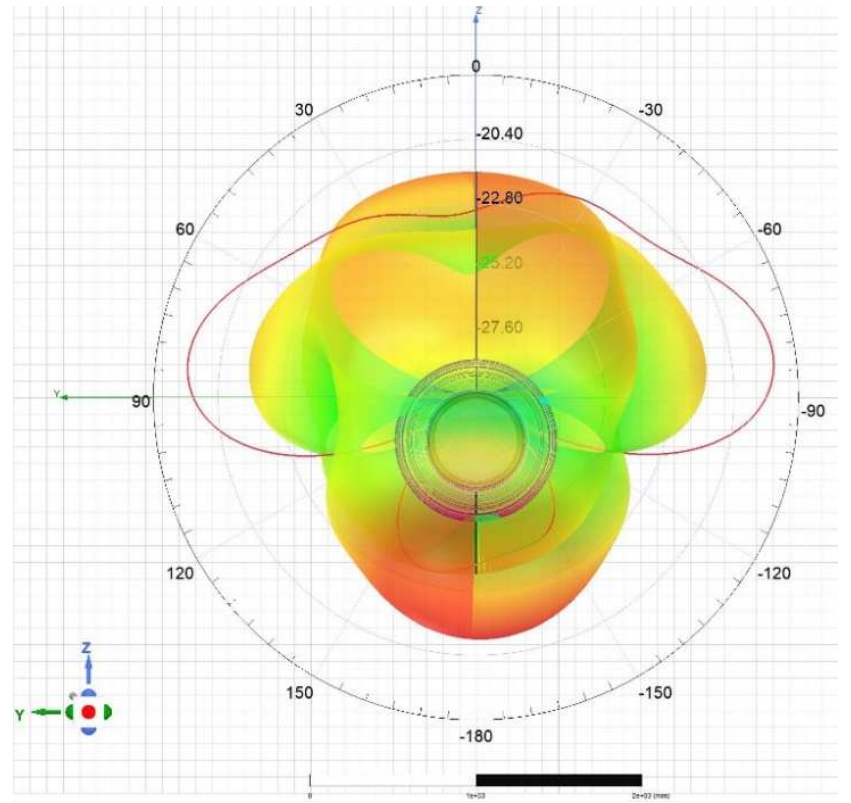

Figure 10: Propagation pattern along the $Y$ and $Z$ axis with the Tire and Wheel in conventional TPMS

\section{CONCLUSION}

Conventional TPMS systems using High Frequency Far-Field RF communication are inefficient in heavy commercial vehicles. In this paper, we leverage on Near-field (NF) communication to provide better signal propagation in harsh environments with high density of metals and liquids faced in heavy commercial vehicle. We simulated the proposed method in the application environment, and we demonstrated that NF-bases solution is less affected by the structure attenuation, providing enhanced propagation distance while consuming less than a quarter of the power level of the conventional TPMS. The low radiating electric energy also reduces the intrinsic safety and interference hazards. We have demonstrated also how NF communication is more resilient around the truck wheel and tire combination. The reduced energy consumption can significantly increase service life of the TPMS sensors in comparison to conventional TPMS. The antenna system is less sensitive to detuning, and would require less matching to the application environment, and so changes to the wheel and tire type, shape or size may tolerable than conventional systems. This and other aspects will be the objective of our future research.

\section{ACKNOWLEDGMENT}

This work was undertaken under the NF Tires project 12513327, funded by the National Research Fund Luxembourg (FNR).

\section{REFERENCES}

[1] R. Steffen and J. Preißinger, "Near Field Communication (NFC) in an Automotive Environment" Second International Workshop on Near Field Communication (2010)
[2] Covic, Grant \& Boys, John \& Budhia, Mickel \& C-Y, Huang. Electric Vehicles - Personal transportation for the future. World Electric Vehicle Journal WEVA. 4. 693-704. 10.3390/wevj4040693 (2011).

[3] Daimler AG, (2017) "Device and method for controlling an access authorisation and/or driving authorisation for a vehicle" US9779564B2.

[4] The Economic commission of European Union, "Regulation number 64 for Uniform provisions concerning the approval of vehicles with regard to their equipment which may include: a temporary-use spare unit, run-flat tyres and/or a run-flat system, and/or a tyre pressure monitoring system," 2010. [Online]. Available: https://op.europa.eu/en/publication-detail/-/publication/ccela9e937b7-4973-bb70-d64999ba110c/language-en. [Accessed: 2-Jan-2021]

[5] N. Q. Dinh, N. Michishita, Y. Yamada, and K. Nakatani, "Electrical characteristics of a very small normal mode helical antenna mounted on a wheel in the TPMS application," Proc. of IEEE International Symposium on Antennas and Propagation, Charleston, SC, June 2009, pp. 1-4.

[6] Continental Tires GmbH, "ContiPressurcheck," 2021. [Online]. Available:https://www.continentaltires.com/transport/products/overvi ew-product-lines/contipressurecheck/about. [Accessed: 2-Jan-2021]

[7] Rida Tawakuli, A, Soua, R., Engel, T. "Evaluation of TPMS Signal Propagation in a Heavy Commercial Vehicle Environement.", https://orbilu.uni.lu/handle/10993/47051, [Accessed: 08-May-2021]

[8] Goodyear Proactive Solutions, "Proactive Tire," 2021. [Online]. Available: https://www.proactive.goodyear.com/solution/goodyeartpms/. [Accessed: 2-Jan-2021]

[9] U.S. Tire manufacturers association "Care and service of commercial truck and bus tires" Maintenance manual [accessed: 2-Jan-2021]

[10] D.Pozar. Microwave Engineering, third edition, John Wiley \& Sons, 2004.

[11] Agbinya, Johnson I.. Principles of Inductive near Field Communications for Internet of Things, River Publishers, 2011

[12] Finkenzeller, Klaus (2003). RFID Handbook: Fundamentals and Applications in Contactless Smart Cards and Identification.2nd Ed. Chichester, England: John Wiley \& Sons Ltd. 446p. ISBN: 0-47084402-7

[13] Chapps, C. \& Delphi Automotive (2001) Near Field or Far Field.

[14] Schantz, H.G. "A real time location system using Near-field electromagnetic ranging", IEEE Antennas and Propagation Society International Symposium, Honolulu, HI, USA ( 2007).

[15] Rida, A. (2016) "Evaluation of RuBee technology applications in steel terminal environment," 21st Logistics Research Network Annual Conference, 2016.

[16] Ansys.Inc, "Maxwell: Low Frequency Electromagnetic Field Simulation," 2021. [Online]. Available: https://www.ansys.com/products/electronics/ansys-maxwell [Accessed: 14-Mar-2021]

[17] European Telecommunications Standards Institute "Electromagnetic compatibility and Radio spectrum Matters (ERM); Short Range Devices (SRD); Radio equipment in the frequency range $9 \mathrm{kHz}$ to 25 $\mathrm{MHz}$ and inductive loop systems in the frequency range $9 \mathrm{kHz}$ to 30 MHz" EUROPEAN STANDARD ETSI EN 300330 [accessed: 14Mar-2021]

[18] A. Özdemirli, M. M. Bilgic and K. Yegin, "TPMS receive antenna design for large vehicles," 2011 XXXth URSI General Assembly and Scientific Symposium, Istanbul, 2011.

[19] H. Zeng and T. Hubing, "The effect of the vehicle body on EM propagation in tire pressure monitoring systems," IEEE Trans. on Antennas and Propagation, vol. 60, no. 8, Aug. 2012, pp. 3941-3949

[20] Dinh, N. Q. et al. "FEKO-based method for electromagnetic simulation of carcass wires embedded in vehicle tires." Applied Computational Electromagnetics Society Journal 26 (2011): 217-22 